Article

\title{
On the Shape Differentiability of Objectives: A Lagrangian Approach and the Brinkman Problem
}

\author{
José Rodrigo González Granada ${ }^{1}$, Joachim Gwinner ${ }^{2}$ (I) and Victor A. Kovtunenko ${ }^{3, *,+}$ (i) \\ 1 Department of Mathematics, Universidad Tecnológica de Pereira, 660003 Pereira, Colombia; \\ jorodryy@utp.edu.co \\ 2 Fakultät für Luft- und Raumfahrttechnik, Institut für Mathematik und Rechneranwendung, \\ Universität der Bundeswehr München, Werner-Heisenberg-Weg 39, 85577 Neubiberg, Germany; \\ joachim.gwinner@unibw-muenchen.de \\ 3 Lavrentyev Institute of Hydrodynamics, Siberian Division of the Russian Academy of Sciences, \\ Novosibirsk 630090, Russia \\ * Correspondence: victor.kovtunenko@uni-graz.at \\ † Current address: Institute for Mathematics and Scientific Computing, Karl-Franzens University of Graz, \\ NAWI Graz, Heinrichstr, 36, 8010 Graz, Austria.
}

Received: 26 September 2018; Accepted: 25 October 2018; Published: 27 October 2018

\begin{abstract}
This paper establishes the shape derivative of geometry-dependent objective functions for use in constrained variational problems. Using a Lagrangian approach, our differentiablity result is based on the theorem of Delfour-Zolésio on directional derivatives with respect to a parameter of shape perturbation. As the key issue of the paper, we analyze the bijection under the kinematic transport of geometries that is needed for function spaces and feasible sets involved in variational problems. Our abstract theoretical result is applied to the Brinkman flow problem under incompressibility and mixed Dirichlet-Neumann boundary conditions, and provides an analytic formula of the shape derivative based on the velocity method.
\end{abstract}

Keywords: constrained optimization; variational inequality; Lagrangian; geometry-dependent objective function; shape derivative; Delfour-Zolésio theorem; divergence-free Brinkman flow

MSC: 49Q10; 49N15; 35Q93

\section{Introduction}

Within an abstract theoretical framework, this paper is devoted to the shape differentiablity of geometry-dependent objective functions as they are considered in constrained variational problems.

Constraints arise in a variety of applications. The constraint operator (see (3)) may become: the trace operator under contact conditions [1-3], the jump operator for cracks and anticracks [4-6], the gradient operator in plasticity [7], the divergence operator under incompressibility conditions [8-10], a state-constraint in mathematical programs with equilibrium constraints [11,12], and the like. The constraint problems are related to parameter identification problems (see the theory in References [13-15] and application to biological systems in Reference [16]), to inverse problems by the mean of observation data used in mathematical physics $[17,18]$ and in acoustics [19-21], to overdetermined and free-boundary problems [22,23]. As an application, in the current paper we focus on the incompressible Brinkman flow problem under a divergence-free condition (see the related modeling of porous medium in References [24,25], well-posedness analysis in Reference [26], and fluid-porous coupling with numerics in References $[27,28])$.

For a general theory of (nonlinear) optimization in infinite dimensions we refer to References [7,29-32] for methods of shape optimization and, in particular, shape derivatives. Specifically in fracture 
mechanics, the shape derivative of energy functionals with respect to a crack extension is called the energy release rate and is of primary importance to engineers as a fracture criterion [33]. In References [4,7], a nonlinear theory of cracks subject to contact conditions and their shape derivatives was developed. Shape perturbation needs a bijective property for function spaces and feasible sets, which, however, fails for curvilinear cracks being in contact. To overcome this obstacle, References $[5,6]$ suggest a $\Gamma$-type convergence, and References $[34,35]$ propose the use of primal-dual Lagrangian reformulation of the crack problems. In incompressible fluid dynamics, to treat the divergence-free condition, divergence-preserving Piola transformation was employed in Reference [10], but lacking a mathematical foundation. The Lagrangian approach to shape optimization was developed further for abstract quadratic objective functions, and the direct proof of shape differentiability was given in Reference [36]. In Reference [9], we applied the primal-dual technique to the Stokes problem under a divergence-free constraint.

In the current work, we extend the Lagrangian approach to abstract geometry-dependent objective functions based on the theorem of Delfour-Zolésio. Thus, we provide the directional derivative with respect to a parameter of shape perturbation in a general setting. We stress that, while bijection fails for the primal cone (a feasible set of primal variables), we obtained the shape derivative in virtue of the bijection property of the dual cone (a feasible set of dual variables). In Section 2, we set a geometry-dependent constrained optimization problem in abstract form, and its shape derivative is derived in Section 3. We applied our theory to a Brinkman flow problem subject to the divergence-free condition in Section 4 and provide an analytic formula of the shape derivative based on the velocity method.

\section{Geometry-Dependent CO Problem}

For real parameter $t \in I:=\left(t_{0}, t_{1}\right)$ with $t_{0}<t_{1}$, we introduce geometry by a kinematic flow

$$
t \mapsto \Omega_{t} \subset \mathbb{R}^{d}, \quad d \in \mathbb{N} .
$$

For every $t \in I$, let $V\left(\Omega_{t}\right)$ and $H\left(\Omega_{t}\right)$ be Hausdorff topological spaces with dual spaces $V^{\star}\left(\Omega_{t}\right)$ and $H^{\star}\left(\Omega_{t}\right)$. Using the order relation for measurable functions in $H\left(\Omega_{t}\right)$, we specify a feasible set provided by the inequality constraint:

$$
K\left(\Omega_{t}\right):=\left\{w \in V\left(\Omega_{t}\right) \mid \quad \mathcal{G}\left(\Omega_{t}\right) w \geq 0\right\}
$$

which is a convex closed cone determined by a linear continuous constraint operator

$$
w \mapsto \mathcal{G}\left(\Omega_{t}\right) w: V\left(\Omega_{t}\right) \mapsto H\left(\Omega_{t}\right)
$$

Extension of Function (3) to a nonlinear operator can be found in Reference [23]. For a geometry-dependent objective given by a continuous and generally nonlinear function

$$
w \mapsto \mathcal{F}\left(w ; \Omega_{t}\right): V\left(\Omega_{t}\right) \mapsto \overline{\mathbb{R}},
$$

we consider the constrained optimization (CO) problem: find $u_{t} \in K\left(\Omega_{t}\right)$ such that

$$
\mathcal{F}\left(u_{t} ; \Omega_{t}\right)=\inf _{w \in K\left(\Omega_{t}\right)} \mathcal{F}\left(w ; \Omega_{t}\right)=: f(t),
$$

where optimal value (OV) function $f: I \mapsto \overline{\mathbb{R}}$. The corresponding solution set implies:

$$
F_{t}:=\left\{u \in K\left(\Omega_{t}\right) \mid \mathcal{F}\left(u ; \Omega_{t}\right)=f(t)\right\}
$$

and may be empty, with exactly one (called a singleton) or more elements. 
Proposition 1. (i) Solution set $F_{t}(6)$ for CO problem (5) is nonempty under the assumption:

(F1) there exists a minimizing sequence $u_{k} \in K\left(\Omega_{t}\right)$, such that $\mathcal{F}\left(u_{k} ; \Omega_{t}\right) \rightarrow f(t)$ as $k \rightarrow \infty$ and an accumulation point $u_{t} \in K\left(\Omega_{t}\right)$ yielding the convergence

$$
u_{k} \rightarrow u_{t} \text { weakly in } V\left(\Omega_{t}\right) \text { as } k \rightarrow \infty
$$

and the lower estimate:

$$
\liminf _{k \rightarrow \infty} \mathcal{F}\left(u_{k} ; \Omega_{t}\right) \geq \mathcal{F}\left(u_{t} ; \Omega_{t}\right)
$$

(ii) If the next assumption holds:

(F2) objective function $u_{t} \mapsto \mathcal{F}\left(\cdot ; \Omega_{t}\right)$ is Gâteaux-differentiable, that is, the following limit exists:

$$
\lim _{s \rightarrow 0} \frac{\mathcal{F}\left(u_{t}+s w ; \Omega_{t}\right)-\mathcal{F}\left(u_{t} ; \Omega_{t}\right)}{s}:=\left\langle\partial_{u} \mathcal{F}\left(u_{t} ; \Omega_{t}\right), w\right\rangle_{\Omega_{t}}, \quad w \in V\left(\Omega_{t}\right)
$$

with $\partial_{u} \mathcal{F}\left(u_{t} ; \Omega_{t}\right) \in V^{\star}\left(\Omega_{t}\right)$ and duality pairing $\langle\cdot, \cdot\rangle_{\Omega_{t}}$ between $V^{\star}\left(\Omega_{t}\right)$ and $V\left(\Omega_{t}\right)$,

then, a first-order optimality condition for CO problem (5) necessitates variational inequality (VI) stated in the form of two relations:

$$
\left\langle\partial_{u} \mathcal{F}\left(u_{t} ; \Omega_{t}\right), u_{t}\right\rangle_{\Omega_{t}}=0, \quad\left\langle\partial_{u} \mathcal{F}\left(u_{t} ; \Omega_{t}\right), w\right\rangle_{\Omega_{t}} \geq 0 \quad \text { for all } w \in K\left(\Omega_{t}\right) .
$$

Conversely, VI (10) is also sufficient for Problem (5) when

(F3) objective function $w \mapsto \mathcal{F}\left(\cdot ; \Omega_{t}\right)$ in (4) is convex.

(iii) Solution set $F_{t}=\left\{u_{t}\right\}$ in (6) is a singleton if the condition (F3) is strict:

(F3') the objective function $w \mapsto \mathcal{F}\left(\cdot ; \Omega_{t}\right)$ in (4) is strictly convex.

Proof. In a reflexive Banach space $V\left(\Omega_{t}\right)$, Condition (7) holds for coercive functions, and Condition (8) is satisfied for the weakly lower semicontinuous functions $w \mapsto \mathcal{F}\left(\cdot ; \Omega_{t}\right)$ in (4). The corresponding proof of assertions (i), (ii), and (iii) is standard, see e.g., (Reference [4] Theorem 1.11).

For objective OV function $f$, we aimed at its shape derivative (SD):

$$
\partial f(t):=\lim _{s \rightarrow 0^{+}} \frac{f(t+s)-f(t)}{s} \quad \text { (one-sided), }
$$

where, according to Problem (5), the perturbed OV is determined over perturbed geometry $\Omega_{t+s}$ :

$$
f(t+s)=\inf _{v \in K\left(\Omega_{t+s}\right)} \mathcal{F}\left(v ; \Omega_{t+s}\right)=\mathcal{F}\left(u_{t+s} ; \Omega_{t+s}\right)
$$

The shape sensitivity of VI was investigated in Reference [32], Chapter 4. However, it can be advantageous to consider a Lagrangian formulation of the problem, see Remark 1.

Let us define the Lagrangian function as $(w, p) \mapsto \mathcal{L}\left(w, p ; \Omega_{t}\right): V\left(\Omega_{t}\right) \times H^{\star}\left(\Omega_{t}\right) \mapsto \overline{\mathbb{R}}$,

$$
\mathcal{L}\left(w, p ; \Omega_{t}\right):=\mathcal{F}\left(w ; \Omega_{t}\right)-\left(p, \mathcal{G}\left(\Omega_{t}\right) w\right) \Omega_{t}
$$

with duality pairing $(\cdot, \cdot)_{\Omega_{t}}$ between $H^{\star}\left(\Omega_{t}\right)$ and $H\left(\Omega_{t}\right)$. For a convex closed cone that is dual to the primal cone (2) (hence called the dual cone):

$$
K^{\star}\left(\Omega_{t}\right):=\left\{p \in H^{\star}\left(\Omega_{t}\right) \mid \quad\left(p, \mathcal{G}\left(\Omega_{t}\right) w\right)_{\Omega_{t}} \geq 0 \text { for all } w \in K\left(\Omega_{t}\right)\right\},
$$


a saddle-point (SP) problem related to Problem (5) reads: find a pair $\left(u_{t}, \lambda_{t}\right) \in V\left(\Omega_{t}\right) \times K^{\star}\left(\Omega_{t}\right)$, such that

$$
\mathcal{L}\left(u_{t}, p ; \Omega_{t}\right) \leq \mathcal{L}\left(u_{t}, \lambda_{t} ; \Omega_{t}\right) \leq \mathcal{L}\left(w, \lambda_{t} ; \Omega_{t}\right) \quad \text { for all }(w, p) \in V\left(\Omega_{t}\right) \times K^{\star}\left(\Omega_{t}\right) .
$$

The Lagrangian OV function $\ell: I \mapsto \overline{\mathbb{R}}$ is defined from the relations

$$
\ell(t)=l_{t}:=\sup _{p \in K^{\star}\left(\Omega_{t}\right)} \inf _{w \in V\left(\Omega_{t}\right)} \mathcal{L}\left(w, p ; \Omega_{t}\right) \leq \inf _{w \in V\left(\Omega_{t}\right)} \sup _{p \in K^{\star}\left(\Omega_{t}\right)} \mathcal{L}\left(w, p ; \Omega_{t}\right)=: l^{t},
$$

and the corresponding solution sets are

$$
V_{t}=\left\{u \in V\left(\Omega_{t}\right) \mid \sup _{p \in K^{\star}\left(\Omega_{t}\right)} \mathcal{L}\left(u, p ; \Omega_{t}\right)=l^{t}\right\}, \quad K_{t}^{\star}=\left\{\lambda \in K^{\star}\left(\Omega_{t}\right) \mid \inf _{w \in V\left(\Omega_{t}\right)} \mathcal{L}\left(w, \lambda ; \Omega_{t}\right)=l_{t}\right\} .
$$

Using Notations (16) and (17), Inequalities (15) determine the set of SP for the Lagrangian (13):

$$
\mathfrak{S}(t):=\left\{\left(u_{t}, \lambda_{t}\right) \in V_{t} \times K_{t}^{\star}, \quad \ell(t)=l_{t}=\mathcal{L}\left(u_{t}, \lambda_{t} ; \Omega_{t}\right)=l^{t}\right\},
$$

which may be empty, a singleton, or contain more than one pair $\left(u_{t}, \lambda_{t}\right)$.

Proposition 2. (i) Under the following assumption:

(G1) mapping $V\left(\Omega_{t}\right) \mapsto H\left(\Omega_{t}\right)$, $w \mapsto \mathcal{G}\left(\Omega_{t}\right) w$ in Problem (3) is surjective,

that is, for every $\zeta \in H\left(\Omega_{t}\right)$ there is at least one $w \in V\left(\Omega_{t}\right)$, such that $\mathcal{G}\left(\Omega_{t}\right) w=\zeta$, the dual cone (14) can be restated equivalently as the cone of non-negative elements in the dual space:

$$
K^{\star}\left(\Omega_{t}\right)=\left\{p \in H^{\star}\left(\Omega_{t}\right) \mid \quad(p, \zeta)_{\Omega_{t}} \geq 0 \quad \text { for all } \zeta \in H\left(\Omega_{t}\right), \zeta \geq 0\right\} .
$$

(ii) Under Assumptions (G1) and (F1), (F2), (F3), the set of SP in (18) is nonempty, and saddle-point $\left(u_{t}, \lambda_{t}\right) \in \mathfrak{S}(t)$ satisfies the optimality system (OS) in the form of variational relations:

$$
\begin{gathered}
\left\langle\partial_{u} \mathcal{F}\left(u_{t} ; \Omega_{t}\right), w\right\rangle_{\Omega_{t}}-\left(\lambda_{t}, \mathcal{G}\left(\Omega_{t}\right) w\right)_{\Omega_{t}}=0 \quad \text { for all } w \in V\left(\Omega_{t}\right), \\
\left(\lambda_{t}, \mathcal{G}\left(\Omega_{t}\right) u_{t}\right)_{\Omega_{t}}=0, \quad\left(p, \mathcal{G}\left(\Omega_{t}\right) u_{t}\right)_{\Omega_{t}} \geq 0 \quad \text { for all } p \in K^{\star}\left(\Omega_{t}\right) .
\end{gathered}
$$

Primal component $u_{t}$ is unique when (F3') holds. Uniqueness of dual component $\lambda_{t}$ takes place if

(G2) the Ladyzhenskaya-Babuška-Brezzi (LBB) condition holds: there exists $\underline{c}>0$, such that

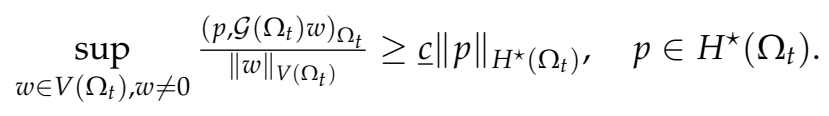

In this case, sets $V_{t}=\left\{u_{t}\right\}$ and $K_{t}^{\star}=\left\{\lambda_{t}\right\}$ in Problem (17) are singletons.

(iii) If a saddle-point in Problem (15) exists, then, under assumption (G1), the primal component $u_{t} \in K\left(\Omega_{t}\right)$ solves the CO problem (5), and the OV functions for objective (5) and for Lagrangian (16) coincide:

$$
f(t)=\ell(t) .
$$

Proof. Assertion (i) is a consequence of the bipolar theorem. In Assertion (ii), using the Proposition 1 solution to the SP problem (15) can be derived in a standard way by determining $u_{t}$ from VI (10) and by setting Lagrange multiplier $\lambda_{t}$ from (20) (e.g., Reference [30] Chapter III, Proposition 3.1). Optimality Conditions (20) and (21) and the uniqueness assertion under LBB Condition (G2) are stated, 
for example, in Reference [3], Theorem 3.14. In Assertion (iii), Problem (5) follows straightforwardly by excluding $\lambda_{t}$ from OS (20) and (21), while Identity (23) is guaranteed by $\left(\lambda_{t}, \mathcal{G}\left(\Omega_{t}\right) u_{t}\right)_{\Omega_{t}}=0$ in $\mathcal{L}\left(u_{t}, \lambda_{t} ; \Omega_{t}\right)$.

Based on Identity (23), the shape derivative of Objective (11) can be substituted by the SD of Lagrangian $\partial \ell$ as follows:

$$
\partial f(t)=\partial \ell(t):=\lim _{s \rightarrow 0^{+}} \frac{\ell(t+s)-\ell(t)}{s} \quad \text { (one-sided). }
$$

According to Definition (16), the perturbed OV $\ell(t+s)=l_{t+s}=\sup _{\mu \in K^{\star}\left(\Omega_{t+s}\right)} \inf _{v \in V\left(\Omega_{t+s}\right)} \mathcal{L}\left(v, \mu ; \Omega_{t+s}\right)$ in (24) is obtained for the perturbed Lagrangian $(v, \mu) \mapsto \mathcal{L}\left(v, \mu ; \Omega_{t+s}\right): V\left(\Omega_{t+s}\right) \times H^{\star}\left(\Omega_{t+s}\right) \mapsto \overline{\mathbb{R}}$,

$$
\mathcal{L}\left(v, \mu ; \Omega_{t+s}\right)=\mathcal{F}\left(v ; \Omega_{t+s}\right)-\left(\mu, \mathcal{G}\left(\Omega_{t+s}\right) v\right)_{\Omega_{t+s}}
$$

and the perturbed SP problem reads: find a pair $\left(u_{t+s}, \lambda_{t+s}\right) \in V\left(\Omega_{t+s}\right) \times K^{\star}\left(\Omega_{t+s}\right)$ such that

$$
\mathcal{L}\left(u_{t+s}, \mu ; \Omega_{t+s}\right) \leq \mathcal{L}\left(u_{t+s}, \lambda_{t+s} ; \Omega_{t+s}\right) \leq \mathcal{L}\left(v, \lambda_{t+s} ; \Omega_{t+s}\right) \text { for all }(v, \mu) \in V\left(\Omega_{t+s}\right) \times K^{\star}\left(\Omega_{t+s}\right) .
$$

However, the theorem of Delfour and Zolésio on differentiability of Lagrangians with respect to parameter $t$ (Reference [31] Chapter 10, Theorem 5.1), which they call the Correa-Seeger theorem, is inapplicable to prove the limit in Problem (24). The reason is that the Lagrangian (25) is determined over geometry-dependent spaces, which, in turn, depend on $s \rightarrow 0^{+}$.

\section{Shape Differentiability of Objectives for CO Problems}

For the above reason, we further parametrized the geometry in Problem (1) as follows: For fixed $t \in I$ let

$$
(s, x) \mapsto \phi_{s}(x),(s, y) \mapsto \phi_{s}^{-1}(y) \in W^{1, \infty}\left(I-t ; W_{\mathrm{loc}}^{1, \infty}\left(\mathbb{R}^{d}\right)\right)^{d}
$$

associate coordinate transformation (CT) $y=\phi_{s}(x)$ and its inverse $x=\phi_{s}^{-1}(y)$ :

$$
\left(\phi_{s}^{-1} \circ \phi_{s}\right)(x)=x, \quad\left(\phi_{s} \circ \phi_{s}^{-1}\right)(y)=y
$$

such that shape perturbation

$$
\Omega_{t+s}=\left\{y \in \mathbb{R}^{d} \mid y=\phi_{s}(x), x \in \Omega_{t}\right\}
$$

builds the diffeomorphism

$$
\phi_{s}: \Omega_{t} \mapsto \Omega_{t+s}, x \mapsto y, \quad \phi_{s}^{-1}: \Omega_{t+s} \mapsto \Omega_{t}, y \mapsto x .
$$

Following the velocity method (e.g., Reference [36]), a given kinematic velocity

$$
\Lambda(t, x) \in L^{\infty}\left(I ; W_{\text {loc }}^{1, \infty}\left(\mathbb{R}^{d}\right)\right)^{d}
$$

establishes Flow (27) by solving the nonautonomous ODE and the transport equation [35]:

$$
\begin{array}{cl}
\frac{d}{d s} \phi_{s}=\Lambda\left(t+s, \phi_{s}\right) \text { for } s>0, & \phi_{s}=x \text { as } s=0 \\
\frac{\partial}{\partial s} \phi_{s}^{-1}+\Lambda(t+s, y) \cdot \nabla_{y} \phi_{s}^{-1}=0 \text { for } s>0, & \phi_{s}^{-1}=y \text { as } s=0 .
\end{array}
$$

Assume that 
(D1) $\operatorname{map}(v, \mu) \mapsto\left(v \circ \phi_{s}, \mu \circ \phi_{s}\right)$ is bijective between the function spaces

$$
V\left(\Omega_{t+s}\right) \mapsto V\left(\Omega_{t}\right), \quad H^{\star}\left(\Omega_{t+s}\right) \mapsto H^{\star}\left(\Omega_{t}\right) ;
$$

(D2) $\operatorname{map} \mu \mapsto \mu \circ \phi_{s}$ is bijective between the dual cones

$$
K^{\star}\left(\Omega_{t+s}\right) \mapsto K^{\star}\left(\Omega_{t}\right)
$$

Assumption (D1) determines the transformed perturbed Lagrangian: $I-t \times V\left(\Omega_{t}\right) \times H^{\star}\left(\Omega_{t}\right) \mapsto \overline{\mathbb{R}}$,

$$
(s, w, p) \mapsto \tilde{\mathcal{L}}\left(s, w, p ; \Omega_{t}\right):=\tilde{\mathcal{F}}\left(s, w ; \Omega_{t}\right)-\left(p, \tilde{\mathcal{G}}\left(s ; \Omega_{t}\right) w\right)_{\Omega_{t}}
$$

with continuous function $(s, w) \mapsto \tilde{\mathcal{F}}\left(s, w ; \Omega_{t}\right): I-t \times V\left(\Omega_{t}\right) \mapsto \overline{\mathbb{R}}$ and continuous operator $(s, w) \mapsto$ $\tilde{\mathcal{G}}\left(s ; \Omega_{t}\right) w: I-t \times V\left(\Omega_{t}\right) \mapsto H\left(\Omega_{t}\right)$, which are resulted from the application of CT (28):

$$
\tilde{\mathcal{F}}\left(s, v \circ \phi_{s} ; \Omega_{t}\right)=\mathcal{F}\left(v ; \Omega_{t+s}\right), \quad\left(\mu \circ \phi_{s}, \tilde{\mathcal{G}}\left(s ; \Omega_{t}\right)\left(v \circ \phi_{s}\right)\right)_{\Omega_{t}}=\left(\mu, \mathcal{G}\left(\Omega_{t+s}\right) v\right)_{\Omega_{t+s}}
$$

in such a way that the identity holds:

$$
\tilde{\mathcal{L}}\left(s, v \circ \phi_{s}, \mu \circ \phi_{s} ; \Omega_{t}\right)=\mathcal{L}\left(v, \mu ; \Omega_{t+s}\right), \quad(v, \mu) \in V\left(\Omega_{t+s}\right) \times H^{\star}\left(\Omega_{t+s}\right) .
$$

According to Problem (37), $\tilde{\mathcal{F}}\left(0, w ; \Omega_{t}\right)=\mathcal{F}\left(w ; \Omega_{t}\right)$ and $\tilde{\mathcal{G}}\left(0 ; \Omega_{t}\right)=\mathcal{G}\left(\Omega_{t}\right)$ as $s=0$.

Remark 1. We remark that Assumption (D2) on the dual cone stated in the (19) form is advantageous in comparison to the condition on Feasible Set (2):

(D2') map $v \mapsto v \circ \phi_{s}$ is bijective between the feasible sets

$$
K\left(\Omega_{t+s}\right) \mapsto K\left(\Omega_{t}\right)
$$

that is needed for OV perturbation for the objective in Problem (12).

In fact, Bijection (D2') fails for integral and derivative-type operators in Feasible Set (2), which are generally not preserved $\mathcal{G}\left(\Omega_{t+s}\right) \circ \phi_{s} \neq \mathcal{G}\left(\Omega_{t}\right)$ under velocity-induced geometry flow $\phi_{s}$ (see the example in Remark 2 ).

Under Assumptions (D1) and (D2), we reset the OV function for the transformed perturbed Lagrangian as $I-t \mapsto \overline{\mathbb{R}}, s \mapsto \tilde{\ell}(\cdot ; t)$ :

$$
\tilde{\ell}(s ; t)=\tilde{l}_{t+s}:=\sup _{p \in K^{\star}\left(\Omega_{t}\right)} \inf _{w \in V\left(\Omega_{t}\right)} \tilde{\mathcal{L}}\left(s, w, p ; \Omega_{t}\right) \leq \inf _{w \in V\left(\Omega_{t}\right)} \sup _{p \in K^{\star}\left(\Omega_{t}\right)} \tilde{\mathcal{L}}\left(s, w, p ; \Omega_{t}\right)=: \tilde{l}^{l+s}
$$

and the corresponding solution sets:

$$
\begin{aligned}
& \tilde{V}_{t+s}=\left\{u \in V\left(\Omega_{t}\right) \mid \sup _{p \in K^{\star}\left(\Omega_{t}\right)} \tilde{\mathcal{L}}\left(s, u, p ; \Omega_{t}\right)=\tilde{l}^{t+s}\right\}, \\
& \tilde{K}_{t+s}^{\star}=\left\{\lambda \in K^{\star}\left(\Omega_{t}\right) \mid \inf _{w \in V\left(\Omega_{t}\right)} \tilde{\mathcal{L}}\left(s, w, \lambda ; \Omega_{t}\right)=\tilde{l}_{t+s}\right\} .
\end{aligned}
$$

If $s=0$, then $\tilde{l}_{t}=l_{t}, \tilde{l}^{l}=l^{l}$ in (16), (40) and $\tilde{V}_{t}=V_{t}, \tilde{K}_{t}^{\star}=K_{t}^{\star}$ for sets (17) and (41) according to (38). Since a solution to Perturbed SP Problem (26) exists, applying Identity (38), we get the solution

$$
\tilde{u}_{t+s}:=u_{t+s} \circ \phi_{s} \in V\left(\Omega_{t}\right), \quad \tilde{\lambda}_{t+s}:=\lambda_{t+s} \circ \phi_{s} \in K^{\star}\left(\Omega_{t}\right)
$$


satisfying the transformed perturbed SP inequalities:

$$
\tilde{\mathcal{L}}\left(s, \tilde{u}_{t+s}, p ; \Omega_{t}\right) \leq \tilde{\mathcal{L}}\left(s, \tilde{u}_{t+s}, \tilde{\lambda}_{t+s} ; \Omega_{t}\right) \leq \tilde{\mathcal{L}}\left(s, w, \tilde{\lambda}_{t+s} ; \Omega_{t}\right) \quad \text { for all }(w, p) \in V\left(\Omega_{t}\right) \times K^{\star}\left(\Omega_{t}\right),
$$

thus, it forms the SP set for transformed perturbed Lagrangian (36) similar to (18):

$$
\tilde{\mathfrak{S}}(t+s):=\left\{\left(\tilde{u}_{t+s}, \tilde{\lambda}_{t+s}\right) \in \tilde{V}_{t+s} \times \tilde{K}_{t+s}^{\star} \mid \quad \tilde{l}_{t+s}=\tilde{\mathcal{L}}\left(s, \tilde{u}_{t+s}, \tilde{\lambda}_{t+s} ; \Omega_{t}\right)=\tilde{l}^{t+s}\right\} .
$$

Following Delfour-Zolésio, we assume that a small $\delta>0$ and a topology in $V\left(\Omega_{t}\right) \times H^{\star}\left(\Omega_{t}\right)$ exist, such that the following hypotheses resulting from the specific representation (36) hold:

(H1) for all $s \in[0, \delta)$, the set of saddle-points $\widetilde{\mathfrak{S}}(t+s)$ in (44) is nonempty;

(H2) for all $u \in \bigcup_{s \in[0, \delta)} \tilde{V}_{t+s} \times \tilde{K}_{t}^{\star}$ and $\tau \in[0, \delta)$, there exists a one-sided partial derivative of the transformed perturbed Lagrangian at perturbation parameter $s=\tau$ in the form:

$$
\frac{\partial}{\partial s} \tilde{\mathcal{L}}\left(\tau, u, p ; \Omega_{t}\right)=\frac{\partial}{\partial s} \tilde{\mathcal{F}}\left(\tau, u ; \Omega_{t}\right)-\left(p, \frac{\partial}{\partial s} \tilde{\mathcal{G}}\left(\tau ; \Omega_{t}\right) u\right)_{\Omega_{t}{ }^{\prime}} \quad p \in H^{\star}\left(\Omega_{t}\right),
$$

obtained from the partial derivatives of the objective and constraint functions as follows

$$
\frac{\partial}{\partial s} \tilde{\mathcal{F}}\left(\tau, u ; \Omega_{t}\right):=\lim _{s \rightarrow 0^{+}} \frac{\tilde{\mathcal{F}}\left(\tau+s, u ; \Omega_{t}\right)-\tilde{\mathcal{F}}\left(\tau, u ; \Omega_{t}\right)}{s}, \quad \frac{\partial}{\partial s} \tilde{\mathcal{G}}\left(\tau ; \Omega_{t}\right):=\lim _{s \rightarrow 0^{+}} \frac{\tilde{\mathcal{G}}\left(\tau+s ; \Omega_{t}\right)-\tilde{\mathcal{G}}\left(\tau ; \Omega_{t}\right)}{s}
$$

(H3) as $s \rightarrow 0^{+}$, accumulation point $\tilde{u}_{t} \in \tilde{V}_{t}$ and subsequence $\tilde{u}_{t+s_{k}} \in \tilde{V}_{t+s_{k}}$ exist, such that

$$
\tilde{u}_{t+s_{k}} \rightarrow \tilde{u}_{t} \quad \text { strongly in } V\left(\Omega_{t}\right) \text { as } s_{k} \rightarrow 0^{+}
$$

and the lower estimate holds:

$$
\liminf _{\tau, s_{k} \rightarrow 0^{+}} \frac{\partial}{\partial s} \tilde{\mathcal{F}}\left(\tau, \tilde{u}_{t+s_{k}} ; \Omega_{t}\right) \geq \frac{\partial}{\partial s} \tilde{\mathcal{F}}\left(0, \tilde{u}_{t} ; \Omega_{t}\right)
$$

(H4) as $s \rightarrow 0^{+}$, accumulation point $\tilde{\lambda}_{t} \in \tilde{K}_{t}^{\star}$ and subsequence $\tilde{\lambda}_{t+s_{k}} \in \tilde{K}_{t+s_{k}}^{\star}$ exist, such that

$$
\tilde{\lambda}_{t+s_{k}} \rightarrow \tilde{\lambda}_{t} \text { strongly in } H^{\star}\left(\Omega_{t}\right) \text { as } s_{k} \rightarrow 0^{+} .
$$

The main result is stated in the next theorem.

Theorem 1. (i) Under Hypotheses (H1)-(H4), accumulation point $\left(\tilde{u}_{t}, \tilde{\lambda}_{t}\right)$ of Sequences (47) and (49) implies an SP of the partial derivative $\frac{\partial}{\partial s} \tilde{\mathcal{L}}\left(0, u, \lambda ; \Omega_{t}\right)$ on $\tilde{V}_{t} \times \tilde{K}_{t}^{\star}$, such that

$$
\sup _{\lambda \in \tilde{K}_{t}^{\star}} \inf _{u \in \tilde{V}_{t}} \frac{\partial}{\partial s} \tilde{\mathcal{L}}\left(0, u, \lambda ; \Omega_{t}\right)=\frac{\partial}{\partial s} \tilde{\mathcal{L}}\left(0, \tilde{u}_{t}, \tilde{\lambda}_{t} ; \Omega_{t}\right)=\inf _{u \in \tilde{V}_{t}} \sup _{\lambda \in \tilde{K}_{t}^{+}} \frac{\partial}{\partial s} \tilde{\mathcal{L}}\left(0, u, \lambda ; \Omega_{t}\right) ;
$$

the shape derivative exists and is represented by the partial derivative at the accumulation point:

$$
\partial_{s} \tilde{\ell}(0 ; t):=\lim _{s \rightarrow 0^{+}} \frac{\tilde{\ell}(s ; t)-\tilde{\ell}(0 ; t)}{s}=\frac{\partial}{\partial s} \tilde{\mathcal{L}}\left(0, \tilde{u}_{t}, \tilde{\lambda}_{t} ; \Omega_{t}\right) \quad \text { (one-sided). }
$$

(ii) Under Assumptions (D1) and (D2), the shape derivatives defined in (24) and (51) coincide:

$$
\partial f(t)=\partial \ell(t)=\partial_{s} \tilde{\ell}(0 ; t) .
$$

Proof. (i) According to Hypothesis (H1), SP inequalities (43) hold for sequence $s_{k} \rightarrow 0^{+}$ from Hypotheses (H3) and (H4). In the next section, we used the accumulation point of 
Sequences (47) and (49) and insert it for a test function $(w, p)=\left(\tilde{u}_{t}, \tilde{\lambda}_{t}\right) \in \tilde{V}_{t} \times \tilde{K}_{t}^{\star} \subset V\left(\Omega_{t}\right) \times K^{\star}\left(\Omega_{t}\right)$ into Problem (43) for $s=s_{k}$ :

$$
\tilde{\mathcal{L}}\left(s_{k}, \tilde{u}_{t+s_{k}}, \tilde{\lambda}_{t} ; \Omega_{t}\right) \leq \tilde{\mathcal{L}}\left(s_{k}, \tilde{u}_{t+s_{k}}, \tilde{\lambda}_{t+s_{k}} ; \Omega_{t}\right) \leq \tilde{\mathcal{L}}\left(s_{k}, \tilde{u}_{t}, \tilde{\lambda}_{t+s_{k}} ; \Omega_{t}\right) .
$$

We also plugged $(w, p)=\left(\tilde{u}_{t+s_{k}}, \tilde{\lambda}_{t+s_{k}}\right) \in \tilde{V}_{t+s_{k}} \times \tilde{K}_{t+s_{k}}^{\star} \subset V\left(\Omega_{t}\right) \times K^{\star}\left(\Omega_{t}\right)$ into (43) as $s=0$ :

$$
\tilde{\mathcal{L}}\left(0, \tilde{u}_{t}, \tilde{\lambda}_{t+s_{k}} ; \Omega_{t}\right) \leq \tilde{\mathcal{L}}\left(0, \tilde{u}_{t}, \tilde{\lambda}_{t} ; \Omega_{t}\right) \leq \tilde{\mathcal{L}}\left(0, \tilde{u}_{t+s_{k}}, \tilde{\lambda}_{t} ; \Omega_{t}\right) .
$$

Subtracting $\tilde{\mathcal{L}}\left(0, \tilde{u}_{t}, \tilde{\lambda}_{t} ; \Omega_{t}\right)$ from left inequality (53), using right inequality (54) and the representation of $\tilde{\mathcal{L}}$ in (36), after division of the result with $s_{k}$ leads to

$$
\begin{aligned}
\frac{\tilde{\mathcal{L}}\left(s_{k}, \tilde{u}_{t+s_{k}}, \tilde{\lambda}_{t+s_{k}} ; \Omega_{t}\right)-\tilde{\mathcal{L}}\left(0, \tilde{u}_{t}, \tilde{\lambda}_{t} ; \Omega_{t}\right)}{s_{k}} \geq \frac{\tilde{\mathcal{L}}\left(s_{k}, \tilde{u}_{t+s_{k}}, \tilde{n}_{t} ; \Omega_{t}\right)-\tilde{\mathcal{L}}\left(0, \tilde{u}_{t+s_{k}}, \tilde{\lambda}_{t} ; \Omega_{t}\right)}{s_{k}}=\frac{\tilde{\mathcal{F}}\left(s_{k}, \tilde{u}_{t+s_{k}} ; \Omega_{t}\right)-\tilde{\mathcal{F}}\left(0, \tilde{u}_{t+s_{k}} ; \Omega_{t}\right)}{s_{k}} \\
-\left(\tilde{\lambda}_{t}, \frac{\tilde{\mathcal{G}}\left(s_{k} ; \Omega_{t}\right)-\tilde{\mathcal{G}}\left(0 ; \Omega_{t}\right)}{s_{k}} \tilde{u}_{t+s_{k}}\right)_{\Omega_{t}}=\frac{\partial}{\partial s} \tilde{\mathcal{F}}\left(\underline{\alpha}_{k} s_{k}, \tilde{u}_{t+s_{k}} ; \Omega_{t}\right)-\left(\tilde{\lambda}_{t}, \frac{\partial}{\partial s} \tilde{\mathcal{G}}\left(\underline{\beta}_{k} s_{k} ; \Omega_{t}\right) \tilde{u}_{t+s_{k}}\right) \Omega_{t},
\end{aligned}
$$

where mean value theorem guarantees existence of weights $\underline{\alpha}_{k}, \underline{\beta}_{k} \in(0,1)$ in (55) due to differentiability property (46). Passing to the limit as $s_{k} \rightarrow 0^{+}$by the virtue of assumptions (47) and (48) proceeds (55) further with the lower estimate:

$$
\begin{aligned}
\liminf _{s_{k} \rightarrow 0^{+}} \frac{\tilde{\mathcal{L}}\left(s_{k}, \tilde{u}_{t+s_{k}}, \tilde{\lambda}_{t+s_{k}} ; \Omega_{t}\right)-\tilde{\mathcal{L}}\left(0, \tilde{u}_{t}, \tilde{\lambda}_{t} ; \Omega_{t}\right)}{s_{k}} \geq \frac{\partial}{\partial s} \tilde{\mathcal{F}}\left(0, \tilde{u}_{t} ; \Omega_{t}\right)-\left(\tilde{\lambda}_{t}, \frac{\partial}{\partial s} \tilde{\mathcal{G}}\left(0 ; \Omega_{t}\right) \tilde{u}_{t}\right)_{\Omega_{t}} & \\
& =\frac{\partial}{\partial s} \tilde{\mathcal{L}}\left(0, \tilde{u}_{t}, \tilde{\lambda}_{t} ; \Omega_{t}\right) .
\end{aligned}
$$

Similarly, subtracting $\tilde{\mathcal{L}}\left(0, \tilde{u}_{t}, \tilde{\lambda}_{t} ; \Omega_{t}\right)$ from right inequality (53) and using left inequality (54) provides the following relations with weights $\bar{\alpha}_{k}, \bar{\beta}_{k} \in(0,1)$ :

$$
\begin{aligned}
\frac{\tilde{\mathcal{L}}\left(s_{k}, \tilde{u}_{t+s_{k}}, \tilde{\lambda}_{t+s_{k}} ; \Omega_{t}\right)-\tilde{\mathcal{L}}\left(0, \tilde{u}_{t}, \tilde{\lambda}_{t} ; \Omega_{t}\right)}{s_{k}} \leq \frac{\tilde{\mathcal{L}}\left(s_{k}, \tilde{u}_{t}, \tilde{t}_{t+s_{k}} ; \Omega_{t}\right)-\tilde{\mathcal{L}}\left(0, \tilde{u}_{t}, \tilde{\lambda}_{t+s_{k}} ; \Omega_{t}\right)}{s_{k}}=\frac{\tilde{\mathcal{F}}\left(s_{k}, \tilde{u}_{t} ; \Omega_{t}\right)-\tilde{\mathcal{F}}\left(0, \tilde{u}_{t} ; \Omega_{t}\right)}{s_{k}} \\
\quad-\left(\tilde{\lambda}_{t+s_{k}}, \frac{\tilde{\mathcal{G}}\left(s_{k} ; \Omega_{t}\right)-\tilde{\mathcal{G}}\left(0 ; \Omega_{t}\right)}{s_{k}} \tilde{u}_{t}\right)_{\Omega_{t}}=\frac{\partial}{\partial s} \tilde{\mathcal{F}}\left(\bar{\alpha}_{k} s_{k}, \tilde{u}_{t} ; \Omega_{t}\right)-\left(\tilde{\lambda}_{t+s_{k}}, \frac{\partial}{\partial s} \tilde{\mathcal{G}}\left(\bar{\beta}_{k} s_{k} ; \Omega_{t}\right) \tilde{u}_{t}\right) \Omega_{t},
\end{aligned}
$$

hence the upper bound

$$
\limsup _{s_{k} \rightarrow 0^{+}} \frac{\tilde{\mathcal{L}}\left(s_{k}, \tilde{u}_{t+s_{k}}, \tilde{\lambda}_{t+s_{k}} ; \Omega_{t}\right)-\tilde{\mathcal{L}}\left(0, \tilde{u}_{t}, \tilde{\lambda}_{t} ; \Omega_{t}\right)}{s_{k}} \leq \frac{\partial}{\partial s} \tilde{\mathcal{L}}\left(0, \tilde{u}_{t}, \tilde{\lambda}_{t} ; \Omega_{t}\right) .
$$

Inequalities (56) and (58) together imply Equality (51) yielding the SD $\partial_{s} \tilde{\ell}$ of OV function $\tilde{\ell}$ given in Problem (40) for transformed Lagrangian $\tilde{\mathcal{L}}$, thus proving Assertion (i).

(ii) When Bijection (34) and (35) holds, from Identity (38) we infer that

$$
\frac{\tilde{\ell}\left(s_{k} ; t\right)-\tilde{\ell}(0 ; t)}{s_{k}}=\frac{\tilde{\mathcal{L}}\left(s_{k}, \tilde{u}_{t+s_{k}}, \tilde{\lambda}_{t+s_{k}} ; \Omega_{t}\right)-\tilde{\mathcal{L}}\left(0, \tilde{u}_{t}, \tilde{\lambda}_{t} ; \Omega_{t}\right)}{s_{k}}=\frac{\mathcal{L}\left(u_{t+s_{k}}, \lambda_{t+s_{k}} ; \Omega_{t+s_{k}}\right)-\mathcal{L}\left(u_{t}, \lambda_{t} ; \Omega_{t}\right)}{s_{k}}=\frac{\ell\left(t+s_{k}\right)-\ell(t)}{s_{k}} .
$$

After passage (59) to the limit as $s_{k} \rightarrow 0^{+}$, this follows the equivalence asserted in (52) between $\partial_{s} \tilde{\ell}$ and the SD $\partial \ell=\partial f$ from (24).

\section{Application to Brinkman Flow}

Let domain $\Omega_{t}$ in Problem (1) have Lipschitz continuous boundary $\partial \Omega_{t}$ obeying the unit normal vector $n^{t}=\left(n_{1}^{t}, \ldots, n_{d}^{t}\right)$ outward to $\Omega_{t}$ and consisting of two nonempty, disjoint sets $\Gamma_{t}^{\mathrm{D}}$ and $\Gamma_{t}^{\mathrm{N}}$. For stationary force $f=\left(f_{1}, \ldots, f_{d}\right) \in H_{\text {loc }}^{1}\left(\mathbb{R}^{d}\right)^{d}$, we consider the Brinkman problem [24]: find a flow velocity $u_{t}=\left(\left(u_{t}\right)_{1}, \ldots,\left(u_{t}\right)_{d}\right)$ and a pressure $\lambda_{t}$ satisfying

$$
-\mu \Delta u_{t}+\alpha u_{t}+\nabla \lambda_{t}=f \quad \text { in } \Omega_{t}
$$




$$
\begin{gathered}
\operatorname{div} u_{t}=0 \quad \text { in } \Omega_{t}, \\
u_{t}=0 \quad \text { on } \Gamma_{t}^{\mathrm{D}}, \quad-\mu \frac{\partial}{\partial n^{t}} u_{t}+\lambda_{t} n^{t}=0 \quad \text { on } \Gamma_{t}^{\mathrm{N}},
\end{gathered}
$$

under mixed Dirichlet-Neumann boundary conditions (62), where parameter $\mu>0$ denotes fluid viscosity, and $\alpha>0$ is the drag coefficient.

Incompressibility Condition (61) determines the operator

$$
\mathcal{G}\left(\Omega_{t}\right)=\operatorname{div}: H_{\Gamma}^{1}\left(\Omega_{t}\right)^{d} \mapsto L^{2}\left(\Omega_{t}\right)
$$

where $L^{2}\left(\Omega_{t}\right)=: H\left(\Omega_{t}\right)=H^{\star}\left(\Omega_{t}\right)$, and Sobolev space $H_{\Gamma}^{1}\left(\Omega_{t}\right)^{d}=: V\left(\Omega_{t}\right)$ accounts for the Dirichlet boundary condition in (62):

$$
H_{\Gamma}^{1}\left(\Omega_{t}\right)^{d}=\left\{w=\left(w_{1}, \ldots, w_{d}\right) \in H^{1}\left(\Omega_{t}\right)^{d} \mid \quad w=0 \text { a.e. } \Gamma_{t}^{\mathrm{D}}\right\} .
$$

The primal cone (2) is presented here by the equality-type constraint:

$$
K\left(\Omega_{t}\right)=\left\{w \in H_{\Gamma}^{1}\left(\Omega_{t}\right)^{d} \mid \quad \operatorname{div} w=0 \text { a.e. } \Omega_{t}\right\} .
$$

Corresponding duality pairings are

$$
\begin{gathered}
\langle u, w\rangle_{\Omega_{t}}=\int_{\Omega_{t}}(\mu \nabla u \cdot \nabla w+\alpha u \cdot w) d x, \quad u, w \in H_{\Gamma}^{1}\left(\Omega_{t}\right)^{d}, \\
\left(p, \mathcal{G}\left(\Omega_{t}\right) w\right)_{\Omega_{t}}=\int_{\Omega_{t}} p \operatorname{div} w d x, \quad p \in L^{2}\left(\Omega_{t}\right),
\end{gathered}
$$

and the latter one builds the dual cone

$$
K^{\star}\left(\Omega_{t}\right)=\left\{p \in L^{2}\left(\Omega_{t}\right) \mid \quad \int_{\Omega_{t}} p \operatorname{div} w d x=0 \quad \text { for all } w \in K\left(\Omega_{t}\right)\right\} .
$$

The underlying objective function in (5) reads:

$$
\mathcal{F}\left(w ; \Omega_{t}\right)=\int_{\Omega_{t}}\left(\frac{\mu}{2}|\nabla w|^{2}+\frac{\alpha}{2}|w|^{2}-f \cdot w\right) d x .
$$

The quadratic function in (69) satisfies Assumptions (F1), (F2), and (F3') of Proposition 1; hence, CO Problem (5) obeys unique solution $u_{t} \in K\left(\Omega_{t}\right)$. The Lagrangian function in (13) takes the form:

$$
\mathcal{L}\left(w, p ; \Omega_{t}\right)=\int_{\Omega_{t}}\left(\frac{\mu}{2}|\nabla w|^{2}+\frac{\alpha}{2}|w|^{2}-f \cdot w-p \operatorname{div} w\right) d x .
$$

If meas $\left(\Gamma_{t}^{\mathrm{N}}\right)>0$, then LBB condition (22) holds, (Reference [3], Theorem 7.2), namely:

$$
\sup _{w \in H_{\Gamma}^{1}\left(\Omega_{t}\right)^{d}, w \neq 0} \frac{\int_{\Omega_{t}} p \operatorname{div} w d x}{\|w\|_{H^{1}\left(\Omega_{t}\right)^{d}}} \geq \underline{c}\|p\|_{L^{2}\left(\Omega_{t}\right)}, \quad \underline{c}>0, \quad p \in L^{2}\left(\Omega_{t}\right) .
$$

This means that the divergence operator in (63) is surjective; thus, Assumptions (G1), (G2) and Assertions (i)-(iii) of Proposition 2 hold true. In the case of equality constraint, the positive cone turns into the whole space $K^{\star}\left(\Omega_{t}\right)=L^{2}\left(\Omega_{t}\right)$ according to (19). OS (20) and (21) implies the solution pair $\left(u_{t}, \lambda_{t}\right) \in H_{\Gamma}^{1}\left(\Omega_{t}\right)^{d} \times L^{2}\left(\Omega_{t}\right)$ satisfying the following variational equations:

$$
\begin{gathered}
\int_{\Omega_{t}}\left(\mu \nabla u_{t} \cdot \nabla w+\left(\alpha u_{t}-f\right) \cdot w-\lambda_{t} \operatorname{div} w\right) d x=0 \quad \text { for all } w \in H_{\Gamma}^{1}\left(\Omega_{t}\right)^{d} \\
\int_{\Omega_{t}} p \operatorname{div} u_{t} d x=0 \quad \text { for all } p \in L^{2}\left(\Omega_{t}\right) .
\end{gathered}
$$


Note that (73) is equivalent to (61) by the fundamental lemma of the calculus of variations, and any smooth solution of (72) after integration by parts leads to Brinkman Equation (60) and the Neumann condition in (62).

Next we apply CT (27). It can be directly checked that bijective Properties (34) and (35), and hence Assumptions (D1) and (D2), hold true. Possible counterexamples are accounted below.

Remark 2. Bijective property (39) in (D2') fails for the primal cone defined in (65) because condition $\operatorname{div}_{y} v=$ $\left(\nabla \phi_{s}^{-T} \circ \phi_{s}\right) \cdot \nabla_{x}\left(v \circ \phi_{s}\right)=0$ is not equivalent to $\operatorname{div}_{x}\left(v \circ \phi_{s}\right)=0$ for $v \in H_{\Gamma}^{1}\left(\Omega_{t+s}\right)^{d}$.

Remark 3. If meas $\left(\Gamma_{t}^{\mathrm{N}}\right)=0$, then the operator in (63) is not surjective since the divergence operator maps $\operatorname{div}: H_{0}^{1}\left(\Omega_{t}\right)^{d} \mapsto L_{0}^{2}\left(\Omega_{t}\right)$, where

$$
L_{0}^{2}\left(\Omega_{t}\right)=\left\{p \in L^{2}\left(\Omega_{t}\right) \mid \int_{\Omega_{t}} p d x=0\right\},
$$

and its topologically dual space $L_{0}^{2}\left(\Omega_{t}\right)^{\star}$ excludes constants. In this case, Bijection (35) in (D2) fails, because $\int_{\Omega_{t+s}} \mu d y=\int_{\Omega_{t}}\left(\mu \circ \phi_{s}\right) \operatorname{det}\left(\nabla \phi_{s}\right) d x=0$ is not equivalent to $\int_{\Omega_{t}}\left(\mu \circ \phi_{s}\right) d x=0$ for $\mu \in L_{0}^{2}\left(\Omega_{t+s}\right)$.

Based on CT $y=\phi_{s}(x)$ with Jacobian matrix $\nabla \phi_{s}$ and its determinant $\operatorname{det}\left(\nabla \phi_{s}\right)>0$, from (70) we get the transformed perturbed objective and Lagrangian according to Relations (36)-(38):

$$
\begin{aligned}
\tilde{\mathcal{L}}\left(s, w, p ; \Omega_{t}\right)=\tilde{\mathcal{F}}\left(s, w ; \Omega_{t}\right)-\int_{\Omega_{t}} p\left(\left(\nabla \phi_{s}^{-T} \circ \phi_{s}\right) \cdot \nabla w\right) \operatorname{det}\left(\nabla \phi_{s}\right) d x \\
\\
\tilde{\mathcal{F}}\left(s, w ; \Omega_{t}\right)=\int_{\Omega_{t}}\left(\frac{\mu}{2}\left|\left(\nabla \phi_{s}^{-T} \circ \phi_{s}\right) \nabla w\right|^{2}+\frac{\alpha}{2}|w|^{2}-\left(f \circ \phi_{s}\right) \cdot w\right) \operatorname{det}\left(\nabla \phi_{s}\right) d x .
\end{aligned}
$$

By checking Hypotheses (H1)-(H4), we prove the result on shape differentiability in the following:

Theorem 2. In the Brinkman problem, there exists the shape derivative expressed by the partial derivative:

$$
\begin{aligned}
\partial f(t)=\partial \ell(t)=\partial_{s} \tilde{\ell}(0 ; t)=\frac{\partial}{\partial s} \tilde{\mathcal{L}}\left(0, u_{t}, \lambda_{t} ; \Omega_{t}\right)=\int_{\Omega_{t}}\left(\frac{\mu}{2} \operatorname{div} \Lambda\left|\nabla u_{t}\right|^{2}-\mu \nabla u_{t} \cdot\left(\nabla \Lambda \nabla u_{t}\right)\right. \\
\\
\left.\quad+\frac{\alpha}{2} \operatorname{div} \Lambda\left|u_{t}\right|^{2}-((\operatorname{div} \Lambda) f+(\Lambda \cdot \nabla) f) \cdot u_{t}+\lambda_{t} \nabla \Lambda^{T} \cdot \nabla u_{t}\right) d x,
\end{aligned}
$$

where $\left(u_{t}, \lambda_{t}\right) \in H_{\Gamma}^{1}\left(\Omega_{t}\right)^{d} \times L^{2}\left(\Omega_{t}\right)$ is the unique SP solving OS (72) and (73), and kinematic velocity $\Lambda(t, x)$ comes from (31).

Proof. (H1) For $s \in[0, \delta)$ with arbitrarily fixed $\delta>0$, the set of SP (44) contains exactly one element:

$$
\tilde{\mathfrak{S}}(t+s)=\left\{\left(\tilde{u}_{t+s}, \tilde{\lambda}_{t+s}\right):=\left(u_{t+s} \circ \phi_{s}, \lambda_{t+s} \circ \phi_{s}\right) \in H_{\Gamma}^{1}\left(\Omega_{t}\right)^{d} \times L^{2}\left(\Omega_{t}\right)\right\}
$$

by transforming solution $\left(u_{t+s}, \lambda_{t+s}\right) \in H_{\Gamma}^{1}\left(\Omega_{t+s}\right)^{d} \times L^{2}\left(\Omega_{t+s}\right)$ to the perturbed at $t+s$ system (72) and (73). This satisfies the transformed perturbed OS for all $(w, p) \in H_{\Gamma}^{1}\left(\Omega_{t}\right)^{d} \times L^{2}\left(\Omega_{t}\right)$ :

$$
\begin{gathered}
\int_{\Omega_{t}}\left(\mu\left(\left(\nabla \phi_{s}^{-T} \circ \phi_{s}\right) \nabla \tilde{u}_{t+s}\right) \cdot\left(\left(\nabla \phi_{s}^{-T} \circ \phi_{s}\right) \nabla w\right)+\right. \\
\quad\left(\alpha \tilde{u}_{t+s}-f \circ \phi_{s}\right) \cdot w \\
\left.-\tilde{\lambda}_{t+s}\left(\nabla \phi_{s}^{-T} \circ \phi_{s}\right) \cdot \nabla w\right) \operatorname{det}\left(\nabla \phi_{s}\right) d x=0, \\
\int_{\Omega_{t}} p\left(\left(\nabla \phi_{s}^{-T} \circ \phi_{s}\right) \cdot \nabla \tilde{u}_{t+s}\right) \operatorname{det}\left(\nabla \phi_{s}\right) d x=0 .
\end{gathered}
$$


(H2) For the velocity $\Lambda$ from (31), we expand as $s \rightarrow 0^{+}$at $(t, x)$ (see Reference [37] Chapter 5):

$$
\nabla \phi_{s}^{-1} \circ \phi_{s}=I-s \nabla \Lambda+\mathrm{o}(s), \quad \operatorname{det}\left(\nabla \phi_{s}\right)=1+s \operatorname{div} \Lambda+\mathrm{o}(s), \quad f \circ \phi_{s}=f+s \Lambda \cdot \nabla f+\mathrm{o}(s)
$$

uniformly over $t$ and $\Omega_{t}$. Plugging (79) into (74) we straightforwardly derived the partial derivative $\frac{\partial}{\partial s} \tilde{\mathcal{L}}\left(0, w, p ; \Omega_{t}\right)$ and extended it to $\frac{\partial}{\partial s} \tilde{\mathcal{L}}\left(\tau, w, p ; \Omega_{t}\right)$ in $(45)$ and $(46)$ for $\tau \in[0, \delta)$ by setting the time-shifted velocity $\left.\Lambda\right|_{t+\tau}:=\Lambda(t+\tau, x)$ instead of $\Lambda=\Lambda(t, x)$. It has the following form:

$$
\begin{aligned}
\frac{\partial}{\partial s} \tilde{\mathcal{L}}\left(\tau, w, p ; \Omega_{t}\right)=\frac{\partial}{\partial s} \tilde{\mathcal{F}}\left(\tau, w ; \Omega_{t}\right)- & \int_{\Omega_{t}} p\left(\left.\operatorname{div} \Lambda\right|_{t+\tau} \operatorname{div} w-\left.\nabla \Lambda\right|_{t+\tau} ^{T} \cdot \nabla w\right) d x \\
\frac{\partial}{\partial s} \tilde{\mathcal{F}}\left(\tau, w ; \Omega_{t}\right)= & \int_{\Omega_{t}}\left(\left.\frac{\mu}{2} \operatorname{div} \Lambda\right|_{t+\tau}|\nabla w|^{2}-\mu \nabla w \cdot\left(\left.\nabla \Lambda\right|_{t+\tau} \nabla w\right)\right. \\
& \left.+\left.\frac{\alpha}{2} \operatorname{div} \Lambda\right|_{t+\tau}|w|^{2}-\left(\left(\left.\operatorname{div} \Lambda\right|_{t+\tau}\right) f+\left(\left.\Lambda\right|_{t+\tau} \cdot \nabla\right) f\right) \cdot w\right) d x
\end{aligned}
$$

(H3) \& (H4) Testing (77) with $w=\tilde{u}_{t+s}$, since $\left(\left(\nabla \phi_{s}^{-T} \circ \phi_{s}\right) \cdot \nabla \tilde{u}_{t+s}\right) \operatorname{det}\left(\nabla \phi_{s}\right)=0$ due to (78), we have

$$
\int_{\Omega_{t}}\left(\mu\left|\left(\nabla \phi_{s}^{-T} \circ \phi_{s}\right) \nabla \tilde{u}_{t+s}\right|^{2}+\alpha\left|\tilde{u}_{t+s}\right|^{2}\right) \operatorname{det}\left(\nabla \phi_{s}\right) d x=\int_{\Omega_{t}}\left(f \circ \phi_{s}\right) \cdot \tilde{u}_{t+s} \operatorname{det}\left(\nabla \phi_{s}\right) d x .
$$

Using the asymptotic expansions (79) and applying Young's inequality with a suitable weight to the right-hand side of (81), this follows the uniform in $s \in[0, \delta)$ estimate:

$$
\left\|\tilde{u}_{t+s}\right\|_{H^{1}\left(\Omega_{t}\right)^{d}}^{2} \leq \frac{1}{\min (\mu, \alpha)} \int_{\Omega_{t}}\left(\mu\left|\nabla \tilde{u}_{t+s}\right|^{2}+\alpha\left|\tilde{u}_{t+s}\right|^{2}\right) d x=\mathrm{O}(1) .
$$

We divide (77) with the norm of $w \neq 0$ and apply the Cauchy-Schwarz inequality such that

$$
\begin{array}{r}
\frac{\int_{\Omega_{t}} \tilde{\lambda}_{t+s}\left(\left(\nabla \phi_{s}^{-T} \circ \phi_{s}\right) \cdot \nabla w\right) \operatorname{det}\left(\nabla \phi_{s}\right) d x}{\|w\|_{H^{1}\left(\Omega_{t}\right)^{d}}}=\frac{\int_{\Omega_{t}}\left(\mu\left(\left(\nabla \phi_{s}^{-T} \circ \phi_{s}\right) \nabla \tilde{u}_{t+s}\right) \cdot\left(\left(\nabla \phi_{s}^{-T} \circ \phi_{s}\right) \nabla w\right)+\left(\alpha \tilde{u}_{t+s}-f \circ \phi_{s}\right) \cdot w\right) \operatorname{det}\left(\nabla \phi_{s}\right) d x}{\|w\|_{H^{1}\left(\Omega_{t}\right)^{d}}} \\
=\mathrm{O}\left(\left\|\tilde{u}_{t+s}\right\|_{H^{1}\left(\Omega_{t}\right)^{d}}+\|f\|_{L^{2}\left(\Omega_{t}\right)^{d}}\right) .
\end{array}
$$

Taking the supremum in (83) over admissible $w$, in the virtue of (82) and LBB condition (71), we get:

$$
\left\|\tilde{\lambda}_{t+s}\right\|_{L^{2}\left(\Omega_{t}\right)}=\mathrm{O}(1) .
$$

By the reflexivity of the underlying function spaces, from (82) and (84) it follows that there exists an accumulation point $\left(\tilde{u}_{t}, \tilde{\lambda}_{t}\right)$ and subsequence $\left(\tilde{u}_{t+s_{k}}, \tilde{\lambda}_{t+s_{k}}\right)$, such that

$$
\left(\tilde{u}_{t+s_{k}}, \tilde{\lambda}_{t+s_{k}}\right) \rightarrow\left(\tilde{u}_{t}, \tilde{\lambda}_{t}\right) \quad \text { weakly in } H_{\Gamma}^{1}\left(\Omega_{t}\right)^{d} \times L^{2}\left(\Omega_{t}\right) \text { as } s_{k} \rightarrow 0^{+} .
$$

From asymptotic Relations (79) and (85), we get the limit of the system of linear equations (77) and (78) in the form of OS (72) and (73). Then, due to the uniqueness of its solution, the accumulation point implies

$$
\left(\tilde{u}_{t}, \tilde{\lambda}_{t}\right)=\left(u_{t}, \lambda_{t}\right)
$$

Using Representations (70), (72), (73), and algebra formula $\frac{1}{2}(a-b)^{2}=-(a-b) b+\frac{1}{2} a^{2}-\frac{1}{2} b^{2}$ we rearrange the following terms:

$$
\begin{array}{r}
\int_{\Omega_{t}}\left(\frac{\mu}{2}\left|\nabla\left(\tilde{u}_{t+s_{k}}-u_{t}\right)\right|^{2}+\frac{\alpha}{2}\left|\tilde{u}_{t+s_{k}}-u_{t}\right|^{2}\right) d x=-\int_{\Omega_{t}}\left(\mu \nabla\left(\tilde{u}_{t+s_{k}}-u_{t}\right) \cdot \nabla u_{t}+\alpha\left(\tilde{u}_{t+s_{k}}-u_{t}\right) \cdot u_{t}\right. \\
\left.+f \cdot\left(\tilde{u}_{t+s_{k}}-u_{t}\right)+\tilde{\lambda}_{t+s_{k}} \operatorname{div} \tilde{u}_{t+s_{k}}\right) d x+\mathcal{L}\left(\tilde{u}_{t+s_{k}}, \tilde{\lambda}_{t+s_{k}} ; \Omega_{t}\right)-\mathcal{L}\left(u_{t}, \tilde{\lambda}_{t+s_{k}} ; \Omega_{t}\right) .
\end{array}
$$


In the virtue of asymptotic Formula (79), from (74) and (43), with test function $w=u_{t}$, it follows

$$
\mathcal{L}\left(\tilde{u}_{t+s_{k}}, \tilde{\lambda}_{t+s_{k}} ; \Omega_{t}\right)-\mathcal{L}\left(u_{t}, \tilde{\lambda}_{t+s_{k}} ; \Omega_{t}\right) \leq \tilde{\mathcal{L}}\left(s_{k}, \tilde{u}_{t+s_{k}}, \tilde{\lambda}_{t+s_{k}} ; \Omega_{t}\right)-\tilde{\mathcal{L}}\left(s_{k}, u_{t}, \tilde{\lambda}_{t+s_{k}} ; \Omega_{t}\right)+c s_{k} \leq c s_{k}
$$

with some constant $c>0$, and $\operatorname{div} \tilde{u}_{t+s_{k}}=\mathrm{O}\left(s_{k}\right)$ according to (78), hence (87) yields that

$$
\limsup _{s_{k} \rightarrow 0^{+}}\left\|\tilde{u}_{t+s_{k}}-u_{t}\right\|_{H^{1}\left(\Omega_{t}\right)^{d}}^{2} \leq 0 .
$$

Subtracting (72) from (77), due to (79) and (88) we have the asymptotic equality

$$
\int_{\Omega_{t}}\left(\tilde{\lambda}_{t+s_{k}}-\lambda_{t}\right) \operatorname{div} w d x=\int_{\Omega_{t}}\left(\mu \nabla\left(\tilde{u}_{t+s_{k}}-u_{t}\right) \cdot \nabla w+\alpha\left(\tilde{u}_{t+s_{k}}-u_{t}\right) \cdot w\right) d x+\mathrm{O}\left(s_{k}\right) .
$$

Therefore, using (89) together with (88), the convergence in (85) is updated to a strong one:

$$
\left(\tilde{u}_{t+s_{k}}, \tilde{\lambda}_{t+s_{k}}\right) \rightarrow\left(\tilde{u}_{t}, \tilde{\lambda}_{t}\right) \text { strongly in } H_{\Gamma}^{1}\left(\Omega_{t}\right)^{d} \times L^{2}\left(\Omega_{t}\right) \text { as } s_{k} \rightarrow 0^{+},
$$

implying (47) and (49) in Hypotheses (H3) and (H4).

Finally, due to the continuity of mapping $(\tau, w) \mapsto \frac{\partial}{\partial s} \tilde{\mathcal{F}}\left(\tau, w ; \Omega_{t}\right)$ in (80) and using the strong Convergence (90), we have:

$$
\lim _{\tau, s_{k} \rightarrow 0^{+}} \frac{\partial}{\partial s} \tilde{\mathcal{F}}\left(\tau, \tilde{u}_{t+s_{k}} ; \Omega_{t}\right)=\frac{\partial}{\partial s} \tilde{\mathcal{F}}\left(0, u_{t} ; \Omega_{t}\right),
$$

that proves (48). Applying Theorem 1 , from Formula (80) at $\tau=0$ with $(w, p)=\left(u_{t}, \lambda_{t}\right)$ and using $\operatorname{div} u_{t}=0$ it follows Formula (75) of the shape derivative and finishes the proof.

We remark a singularity of the mixed Dirichlet-Neumann boundary value Problem (72) and (73) at intersection $\overline{\Gamma_{t}^{\mathrm{D}}} \cap \overline{\Gamma_{t}^{\mathrm{N}}}$, such that its solution $\left(u_{t}, \lambda_{t}\right)$ is generally not in $H^{2}\left(\Omega_{t}\right)^{d} \times H^{1}\left(\Omega_{t}\right)$. Therefore:

Corollary 1. Let the singular set be localized in a domain $\overline{\omega_{t}} \subset \overline{\Omega_{t}}$ such that the solution $\left(u_{t}, \lambda_{t}\right)$ of (72) and (73) $\left(u_{t}, \lambda_{t}\right) \in H^{2}\left(\Omega_{t} \backslash \omega_{t}\right)^{d} \times H^{1}\left(\Omega_{t} \backslash \omega_{t}\right)$, force $f \equiv$ const and velocity $\Lambda \equiv$ const in $\omega_{t}$. In this case, using integration of (75) by parts, the following Hadamard representation over the boundary of $\Omega_{t} \backslash \omega_{t}$ with the outward normal $n^{t}$ holds true:

$$
\frac{\partial}{\partial s} \tilde{\mathcal{L}}\left(0, u_{t}, \lambda_{t} ; \Omega_{t}\right)=\int_{\partial\left(\Omega_{t} \backslash \omega_{t}\right)} \Lambda \cdot\left(n^{t}\left(\frac{\mu}{2}\left|\nabla u_{t}\right|^{2}+\frac{\alpha}{2}\left|u_{t}\right|^{2}-f \cdot u_{t}\right)-\nabla u_{t}\left(\mu \frac{\partial}{\partial n^{t}} u_{t}-\lambda_{t} n^{t}\right)\right) d S_{x} .
$$

Proof. In domain $D$ with regular boundary $\partial D$ and unit normal vector $n$ outward to $D$, for smooth functions $w$ and $p$, we used the following formulas of integration by parts written component-wisely with the convention of summation over repeated indices $i, j, k=1, \ldots, d$ :

$$
\begin{gathered}
\int_{D}\left(\frac{1}{2} \Lambda_{k, k} w_{i, j}^{2}-w_{i, k} \Lambda_{k, j} w_{i, j}\right) d x=\int_{D} \Lambda_{k} w_{i, j j} w_{i, k} d x+\int_{\partial D}\left(\Lambda_{k} n_{k} \frac{1}{2} w_{i, j}^{2}-\Lambda_{k} w_{i, k} w_{i, j} n_{j}\right) d S_{x} \\
\int_{D} \frac{1}{2} \Lambda_{k, k} w_{i}^{2} d x=-\int_{D} \Lambda_{k} w_{i, k} w_{i} d x+\int_{\partial D} \Lambda_{k} n_{k} \frac{1}{2} w_{i}^{2} d S_{x} \\
-\int_{D}\left(\Lambda_{k, k} f_{i}+\Lambda_{k} f_{i, k}\right) w_{i} d x=\int_{D} \Lambda_{k} f_{i} w_{i, k} d x-\int_{\partial D} \Lambda_{k} n_{k} f_{i} w_{i} d S_{x} \\
\int_{D} p \Lambda_{k, i} w_{i, k} d x=-\int_{D} \Lambda_{k}\left(p_{i} w_{i, k}+p w_{i, i k}\right) d x+\int_{\partial D} \Lambda_{k} w_{i, k} p n_{i} d S_{x} .
\end{gathered}
$$


The summation of four equations in (93) (where the first equation is multiplied with $\mu$ and the second one with $\alpha$ ) provides the identity

$$
\begin{aligned}
& \int_{D}\left(\frac{\mu}{2} \operatorname{div} \Lambda|\nabla w|^{2}-\mu \nabla w \cdot\right.\left.(\nabla \Lambda \nabla w)+\frac{\alpha}{2} \operatorname{div} \Lambda|w|^{2}-((\operatorname{div} \Lambda) f+(\Lambda \cdot \nabla) f) \cdot w+p \nabla \Lambda^{T} \cdot \nabla w\right) d x \\
&=\int_{D}((\Lambda \cdot w)(\mu \Delta w-\alpha w+f-\nabla p)-p \Lambda \cdot \operatorname{div} w) d x \\
& \quad+\int_{\partial D} \Lambda \cdot\left(n\left(\frac{\mu}{2}\left|\nabla u_{t}\right|^{2}+\frac{\alpha}{2}|w|^{2}-f \cdot w\right)-\nabla w\left(\mu \frac{\partial}{\partial n} w-p n\right)\right) d S_{x} .
\end{aligned}
$$

The integral in (75) over $\omega_{t}$ vanishes when $\nabla f \equiv 0$ and $\nabla \Lambda \equiv 0$ here. For the complement integral over $\Omega_{t} \backslash \omega_{t}$, we applied Formula (94) and Equations (60), (61) to derive (92). The proof is completed.

Remark 4. If the parameter $\alpha=0$, then (60) turns into the Stokes flow equation. In this case, the shape differentiability result of Theorem 2 holds true for the Stokes problem and was proved by direct method in the earlier work [9].

Remark 5. If parameter $\mu=0$, then (60) turns into the equation describing Darcy flow [16]. In this case, function space $V\left(\Omega_{t}\right)=\left\{w \in H\left(\operatorname{div} ; \Omega_{t}\right) \mid w=0\right.$ a.e. $\left.\Gamma_{t}^{\mathrm{D}}\right\}$, where

$$
H\left(\operatorname{div} ; \Omega_{t}\right)=\left\{w=\left(w_{1}, \ldots, w_{d}\right) \in L^{2}\left(\Omega_{t}\right)^{d} \mid \quad \operatorname{div} w \in L^{2}\left(\Omega_{t}\right)\right\} .
$$

It fails bijection property (34) in (D1), because $\operatorname{div}_{y} v=\left(\nabla \phi_{s}^{-T} \circ \phi_{s}\right) \cdot \nabla_{x}\left(v \circ \phi_{s}\right) \in L^{2}\left(\Omega_{t}\right)$ does not imply $\operatorname{div}_{x}\left(v \circ \phi_{s}\right) \in L^{2}\left(\Omega_{t}\right)$ and vice versa (see also Remark 2 ).

\section{Conclusions}

The current study extends the class of shape differential models to the incompressible Brinkman flow problem under mixed Dirichlet-Neumann boundary conditions. This result can be continued to flow problems under pure no-slip boundary conditions for area-preserving maps within $L_{0}^{2}\left(\Omega_{t}\right)$-spaces (see Remark 3) that form special linear group $\mathrm{SL}(d)$ in $\mathbb{R}^{d}$. Future research in this field might use divergence-preserving maps (see Remark 2 and Remark 5) like Piola transformation, which challenges a rigorous mathematical tool. The other challenging direction concerns examining the geometric singularity of the mixed Dirichlet-Neumann boundary value problem (72) and (73) at intersection point $\overline{\Gamma_{t}^{\mathrm{D}}} \cap \overline{\Gamma_{t}^{\mathrm{N}}}$.

Author Contributions: Supervision, J.G.; Project Administration, V.A.K.; Funding Acquisition, J.R.G.G.

Funding: This research was funded by the Austrian Science Fund (FWF) grant number P26147-N26: "Object identification problems: numerical analysis" (PION).

Acknowledgments: V.A.K. thanks the Austrian Academy of Sciences (OeAW) for their partial support, the Colombian Institute for Education and Technical Studies Abroad (ICETEX), and Universidad Tecnológica de Pereira (UTP) for supporting his visit of UTP and the Conference ICAMI 2017 at San Andrés Island, Colombia; and the Chair of the Conference Organizing Committee O.O. Vasilieva for their help.

Conflicts of Interest: The authors declare no conflict of interest.

\section{Abbreviations}

The following abbreviations are used in this manuscript:

$\begin{array}{ll}\text { CO } & \text { constrained optimization } \\ \text { CT } & \text { coordinate transformation } \\ \text { LBB } & \text { Ladyzhenskaya-Babuška-Brezzi } \\ \text { ODE } & \text { ordinary differential equation } \\ \text { OS } & \text { optimality system } \\ \text { OV } & \text { optimal value }\end{array}$


SD shape derivative

SP saddle-point

VI variational inequality

\section{References}

1. Gwinner, J.; Ovcharova, N. From solvability and approximation of variational inequalities to solution of nondifferentiable optimization problems in contact mechanics. Optimization 2015, 64, 1683-1702. [CrossRef]

2. Gwinner, J.; Stephan, E.P. Advanced Boundary Element Methods: Treatment of Boundary Value, Transmission and Contact Problems; Springer: Berlin, Germany, 2018; ISBN 978-3-319-92000-9.

3. Kikuchi, N.; Oden, J.T. Contact Problems in Elasticity: A Study of Variational Inequalities and Finite Element Methods; SIAM: Philadelphia, PA, USA, 1988; ISBN 978-0-8987-1468-5.

4. Khludnev, A.M.; Kovtunenko, V.A. Analysis of Cracks in Solids; WIT-Press: Southampton, UK, 2000; ISBN 978-1-8531-2625-3.

5. Lazarev, N.P.; Rudoy, E.M. Shape sensitivity analysis of Timoshenko's plate with a crack under the nonpenetration condition. Z. Angew. Math. Mech. 2014, 94, 730-739. [CrossRef]

6. Shcherbakov, V.V. Shape derivative of the energy functional for the bending of elastic plates with thin defects. J. Phys. Conf. Ser. 2017, 894, 012084. [CrossRef]

7. Khludnev, A.M.; Sokolowski, J. Modelling and Control in Solid Mechanics; Birkhäuser: Basel, Switzerland, 1997; ISBN 978-3-0348-8984-1.

8. Chesnokov, A.A.; Kovtunenko, P.V. Weak discontinuities in solutions of long-wave equations for viscous flow. Stud. Appl. Math. 2014, 132, 50-64. [CrossRef]

9. Kovtunenko, V.A.; Ohtsuka, K. Shape differentiability of Lagrangians and application to Stokes problem. SIAM J. Control Optim. 2018, 56, 3668-3684. [CrossRef]

10. Moubachir, M.; Zolésio, J.-P. Moving Shape Analysis and Control; Chapman \& Hall/CRC: Boca Raton, FL, USA, 2006; ISBN 978-1-5848-8611-2.

11. De los Reyes, J.C. On the optimal control of some nonsmooth distributed parameter systems arising in mechanics. GAMM-Mitteilungen 2018, 40, 268-286. [CrossRef]

12. Hintermüller, M.; Surowiec, T. First-order optimality conditions for elliptic mathematical programs with equilibrium constraints via variational analysis. SIAM J. Optim. 2011, 21, 1561-1593. [CrossRef]

13. Gwinner, J. On two-coefficient identification in elliptic variational inequalities. Optimization 2018, 67, 1-4. [CrossRef]

14. Gwinner, J.; Jadamba, B.; Khan, A.A.; Sama, M. Identification in variational and quasi-variational inequalities. J. Convex Anal. 2018, 25, 545-569, ISSN 0944-6532.

15. Radwan, A.; Vasilieva, O.; Enkhbat, R.; Griewank, A.; Guddat, J. Parametric approach to optimal control. Optim. Lett. 2012, 6, 1303-1316. [CrossRef]

16. Batzel, J.J.; Bachar, M.; Kappel, F. Mathematical Modeling and Validation in Physiology: Applications to the Cardiovascular and Respiratory Systems; Springer: Berlin/Heidelberg, Germany, 2012; ISBN 978-3-6423-2882-4.

17. Hasanov Hasanoğlu, A.; Romanov, V.G. Introduction to Inverse Problems for Differential Equations; Springer: Berlin, Germany, 2017; ISBN 978-3-319-62796-0.

18. Lavrentiev, M.M.; Avdeev, A.V.; Lavrentiev, M.M., Jr.; Priimenko, V.I. Inverse Problems of Mathematical Physics; De Gruyter: Berlin, Germany, 2012; ISBN 978-9-0676-4396-2.

19. Alekseev, G.V. Invisibility Problem in Acoustics Optics and Heat Transfer; Dalnauka: Vladivostok, Russia, 2016; ISBN 978-5-8044-1045-3. (In Russian)

20. Cakoni, F.; Kovtunenko, V.A. Topological optimality condition for the identification of the center of an inhomogeneity. Inverse Probl. 2018, 34, 035009. [CrossRef]

21. Kovtunenko, V.A.; Kunisch, K. High precision identification of an object: Optimality-conditions-based concept of imaging. SIAM J. Control Optim. 2014, 52, 773-796. [CrossRef]

22. Haslinger, J.; Ito, K.; Kozubek, T.; Kunisch, K.; Peichl, G. On the shape derivative for problems of Bernoulli type. Interfaces Free Bound. 2009, 11, 317-330. [CrossRef]

23. Ito, K.; Kunisch, K. Lagrange Multiplier Approach to Variational Problems and Applications; SIAM: Philadelphia, PA, USA, 2008; ISBN 978-0-8987-1649-8. 
24. Hill, A.A.; Rajagopal, K.R.; Vergori, L. On the stability and uniqueness of the flow of a fluid through a porous medium. Z. Angew. Math. Phys. 2016, 67, 49. [CrossRef]

25. Nield, D.; Bejan, A. Convection in Porous Media; Springer: New York, NY, USA, 2006; ISBN 978-0387-29096-6.

26. Kohr, M.; Lanza de Cristoforis, M.; Mikhailov, S.E.; Wendland, W.L. Integral potential method for transmission problem with Lipschitz interface in $\mathbb{R}^{3}$ for the Stokes and Darcy-Forchheimer-Brinkman PDE systems. Z. Angew. Math. Phys. 2016, 67, 116. [CrossRef]

27. Angot, P. Well-posed Stokes/Brinkman and Stokes/Darcy coupling revisited with new jump interface conditions. ESAIM Math. Model. Numer. Anal. 2018. [CrossRef]

28. Bernardi, C.; Hecht, F.; Pironneau, O. Coupling Darcy and Stokes equations for porous media with cracks. ESAIM Math. Model. Numer. Anal. 2005, 39,7-35. [CrossRef]

29. Abello Muñoz, C.A.; Cárdenas Alzate, P.P.; González Granada, J.R. On the coercive functions and minimizers. Adv. Stud. Theor. Phys. 2017, 11, 709-715. [CrossRef]

30. Ekeland, I.; Temam, R. Convex Analysis and Variational Problems; North-Holland: Amsterdam, The Netherlands, 1976; ISBN 978-0-4441-0898-2.

31. Delfour, M.C.; Zolésio, J.-P. Shape and Geometries: Metrics, Analysis, Differential Calculus, and Optimization; SIAM: Philadelphia, PA, USA, 2011; ISBN 978-0-898719-36-9.

32. Sokolowski, J.; Zolésio, J.-P. Introduction to Shape Optimization. Shape Sensitivity Analysis; Springer: Berlin/ Heidelberg, Germany, 1992; ISBN 978-3-6426-3471-0.

33. Kovtunenko, V.A.; Leugering, G. A shape-topological control problem for nonlinear crack - defect interaction: the anti-plane variational model. SIAM J. Control Optim. 2016, 54, 1329-1351. [CrossRef]

34. Kovtunenko, V.A. Primal-dual methods of shape sensitivity analysis for curvilinear cracks with non-penetration. IMA J. Appl. Math. 2006, 71, 635-657. [CrossRef]

35. Kovtunenko, V.A.; Kunisch, K. Problem of crack perturbation based on level sets and velocities. Z. Angew. Math. Mech. 2007, 87, 809-830. [CrossRef]

36. Hintermüller, M.; Kovtunenko, V.A. From shape variation to topology changes in constrained minimization: A velocity method-based concept. Optim. Methods Softw. 2011, 26, 513-532. [CrossRef]

37. Walker, S.W. The Shapes of Things: A Practical Guide to Differential Geometry and the Shape Derivative; SIAM: Philadelphia, PA, USA, 2015; ISBN 978-1-6119-7395-2.

(C) 2018 by the authors. Licensee MDPI, Basel, Switzerland. This article is an open access article distributed under the terms and conditions of the Creative Commons Attribution (CC BY) license (http:/ / creativecommons.org/licenses/by/4.0/). 